\title{
Evaluation of an Information Service for Enhanced Multiaccess Media Delivery
}

\author{
Jukka Mäkelä, Markus Luoto, Tiia Sutinen, Kostas Pentikousis \\ VTT Technical Research Centre of Finland \\ PO Box 1100, Fl-90571 \\ Oulu, Finland \\ firstname.lastname@vtt.fi
}

\begin{abstract}
Multimedia delivery in mobile multiaccess network environments is gaining traction as a key area within the future Internet research domain. When network heterogeneity is coupled with the proliferation of multiaccess capabilities of mobile handheld devices, one can expect new avenues for the development of novel services and applications. In particular, novel mechanisms for audio/video delivery over multiaccess networks may prove to be a major disruptive technology. In this paper we present and evaluate a distributed information service, which can be used to enhance media delivery over multiaccess networks. After describing the information service, which is built upon the mobility triggering framework (TRG) and the distributed control and management framework (DCMF), we introduce our testbed which includes 3G/HSPA, WLAN and WiMAX network accesses. We present results that demonstrate the value of the proposed information service in enhancing video delivery in practice by minimizing service disruption in an involved scenario. Finally, we conclude the paper outlining future work items.
\end{abstract}

\section{Keywords}

Mobility management, multiaccess, event service, information service

\section{INTRODUCTION}

Heterogeneous network environments and multiaccess capability obviously create new opportunities, but also challenges for applications. Especially for Quality of Service (QoS) sensitive multimedia applications, it is difficult to fully utilize the potential of multiaccess networks as host mobility and resulting handovers introduce harmful variability to the QoS characteristics of the underlying network connection. It is also difficult for the applications to introduce sufficient corrective measures (e.g. adaptation) to cope with the handover-induced QoS changes as the current L2/L3 mobility solutions hide mobility completely from higher layers.

Permission to make digital or hard copies of all or part of this work for personal or classroom use is granted without fee provided that copies are not made or distributed for profit or commercial advantage and that copies bear this notice and the full citation on the first page. To copy otherwise, to republish, to post on servers or to redistribute to lists, requires prior specific permission and/or a fee.

Mobimedia'09, September 7-9, 2009, London, UK.

Copyright 2009 ICST 978-963-9799-62-2/00/0004 ...\$5.00.
Moreover, in many cases, the handover decision-making of current mobility protocols is suboptimal for multimedia applications as the decisions are solely based on lower layer information (e.g. RSS measurements) and do not take into account the applications' requirements for network access.

Mobility management in a heterogeneous network needs support from mobility protocols like Mobile IP [1] or Host Identity Protocol [2]. These protocols provide one key mechanism of the mobility management, handover management, that provides the means for changing the point of network attachment. The change of the point of attachment is usually based on a decision made by the mobility protocols or by a separate entity guiding the protocols [7]. This decision is based on the conditions prevailing in the terminal and/or the network side. More specifically, the decision is triggered by an event or events created within the system as a response to a change in the conditions. Typically, a change in the received signal strength, access network availability or user preferences are events leading to handover decisions.

Since conditions are changing both in the terminal's and the networks' side, distributed decision-making is needed for the handover management. For enabling these distributed actions, there is a need for a framework to exchange information between various entities that may be located on the different layers of the protocol stack and in different network nodes. Our approach is to use a distributed information service for collecting and distributing events created by condition changes in the different parts of the system.

The triggering functionality and distributed control and management framework (DCMF) proposed in this paper provide the required cross-layer signalling mechanisms to enable application-aware handover decision-making and enhanced application adaptation in the presence of heterogeneous handovers. First of all, a multimedia application is capable of informing the handover manager about its QoS requirements (bandwidth, delay, etc.). This information can be collected, for example, from application-layer session descriptors (Session Description Protocol) and communicated to the handover manager via TRG. The handover manager can then map this information to that received from lower layers (e.g. Media Independent Handover (MIH) [5]) and from the network to select the best possible access for the multimedia transmission.

In this paper, we concentrate on evaluating and validating our mechanisms for the information service to show not only that we have an implementation of the mechanism already available but also to demonstrate the performance benefits achieved in a mobile multiaccess test case. For the case 
evaluation, we have created a real heterogeneous multiaccess testbed environment with 3G/HSPA, WLAN and WiMAX access technologies. The benefits obtained from using our information service is validated in the context of changing the point of attachment between the different access networks of the testbed environment.

The rest of the paper is structured as follows. Section 2 describes the main concepts of our information system. Section 3 describes the experimental study scenario and shows the evaluation cases with results. Section 4 describes the related work and finally, section 5 concludes the paper.

\section{INFORMATION SYSTEM CONCEPT}

In our previous work [3], visited also in the next subsection, we presented the main concepts and principles of a general framework suitable for multiaccess mobility management. At the core of this framework lies the triggering functional entity (TRG) which is capable of collecting, storing temporarily, and delivering notifications called triggers within an IP-based communications system. Triggers signal a change in the system status and they may originate from virtually any entity that influences node communication. This is in contrast with IEEE 802.21 [5] (and proposals solely based on this emerging standard), in which events originate solely from the lower layers. On the other hand, similarly to IEEE 802.21, TRG leaves the interpretation of the information carried in the triggers to the consumers, which can use the delivered notifications as input to their own decision-making.

We are interested in developing a scalable and distributed system that uses cascaded TRGs to deliver a distributed information service between different network nodes. We recently demonstrated the feasibility of our proposal using cascaded TRGs for delivering triggers from WLAN access points to a Mobile IP client [4]. Cascaded TRG entities, located at various network locations and the mobile nodes, can exchange information and collaborate in order to increase the performance of multiaccess mobile devices. Cascaded TRG can capitalize on the availability of IEEE 802.21 $\mathrm{MIH}$ entities in the network, as both remote and local MIH events can be converted into triggers. As explained in [10], the mechanisms and services introduced by the recently finalized IEEE standard are not sufficient for handover triggering and decision processes. IEEE 802.21 provides a way to command and use the lower layer information to enable seamless handovers and multiaccess, but it does not provide a way to use also upper layer information like user or application preferences as the TRG framework does. That is why we propose that instead of using only the services provided by 802.21 the system should use the TRG framework together with 802.21 services. 802.21 can be for example the source entity that provides the lower layer information for the TRG framework.

Most of the current application adaptation solutions rely on client feedback to make dynamic adaptation decisions (e.g. adjustments to bit rate, frame rate or resolution in the case of a video streaming application) in the server or some intermediate media-aware network node. The feedback for RTP/UDP-based multimedia applications is typically realized using RTCP. Through TRG and DCMF, an adaptive application can gain access to more extensive information (e.g. MIH events, MIP or HIP mobility state changes) that it can use in adapting its operation more efficiently in a mul-
Table 1: Trigger format

\begin{tabular}{|l|c|l|}
\hline $\begin{array}{l}\text { Trigger data } \\
\text { member }\end{array}$ & Type & Description \\
\hline id & integer & $\begin{array}{l}\text { Trigger identifier, same as } \\
\text { producer identifier. Maps } \\
\text { producer name to identifier. }\end{array}$ \\
\hline type & integer & $\begin{array}{l}\text { Specific to the trigger iden- } \\
\text { tifier. Mapping producer in- } \\
\text { formation to type. }\end{array}$ \\
\hline value & std:string & Specific to trigger type. \\
\hline timestamp & time_t & $\begin{array}{l}\text { Time that a trigger enters } \\
\text { the TRG repository. }\end{array}$ \\
\hline
\end{tabular}

tiaccess environment. Cascaded TRGs can potentially be employed also in end-to-end feedback delivery, as described in [20], in cases where traditional feedback protocols do not suffice.

TRG can feed the multiaccess mobility management decisionmaking entities which employ mechanisms such as those presented in [7]. The decision on which interface to use can be defined as a multiple attribute decision making problem and it can be based on different algorithms like Simple Additive Weighting and Multiplicative Exponent Weighting as detailed in papers $[8,9]$. These are just a couple of examples that could be used and the decision making is out of scope of this paper. Other procedures e.g. genetic algorithms for solving the decision problems are also possible as the design of DCMF allows the flexible use of different algorithms.

\subsection{Triggering functional entity}

The TRG functional entity has three major components: event collection, processing and the trigger repository. Event collection receives events from triggering event sources via collection interface. Triggers can be created by implementing the trigger event collection functionality, the event collection interface. This event collection interface allows sources to register their triggers which makes them available to consumers. The implementation of TRG may have several event collectors which are responsible for collecting different types of events. The trigger repository is designed to store the triggers. It is planned to meet the requirements of mobility management, but can be used to store non-mobility triggers as well. Repository's basic primitives include adding, removing, updating, and disseminating triggers in a standardized format (Table 1).

The source of an event can be a hardware device, a system component implemented in kernel space, or an application implemented in user space. For example, each device driver could implement its own event collection functionality, which would be capable of handling triggering events produced by the specific device only. Event sources can also be located in the network such as at active network elements or at the user's home network e.g. in the Mobile IP [1]home agent. The TRG implementation can act also as a consumer or a producer to another TRG located in a different node. Thus, orchestrating the collaboration of, perhaps, several collection entities is needed in order to efficiently gather a larger amount of events.

Having dedicated collectors for different event sources enables the use of TRG in different operating systems as well. The collector can format the events to the format (Table 1) 
that TRG understands and there is no need to modify the core of the TRG functionality; instead the collector can be modified as necessary. This is also one of the key points in the architectural design of TRG that enables it to handle cross-layer information by having collectors at different layers as needed. For example TRG can get similar information regarding the connectivity in FreeBSD through a collector that uses Route Socket and in Linux through a similar collector using RTnetlink socket.

After events are collected from the producers, they are handed over to the trigger processing engine which is responsible for time-stamping and reformatting triggers (if necessary), and assigning them to the appropriate group. Those entities called consumers which are interested in certain triggering events can subscribe by specifying a set of triggers (and, optionally, filtering rules) and are expected to unsubscribe when they do not wish to receive them any longer. For each consumer subscription, TRG makes sure that filters are syntactically correct, and accepts or rejects the subscription. Basic rules can also be used as building blocks for crafting more sophisticated rules. More details about the TRG architecture, functionality and the performance evaluation can be found in [3] and [12].

\subsection{Distributed information service}

For this paper we built a heterogeneous test environment and evaluated the distributed way of using TRG called cascaded triggering. We have also started to define and implement a distributed control and management framework (DCMF) [11] illustrated in Figure 1.

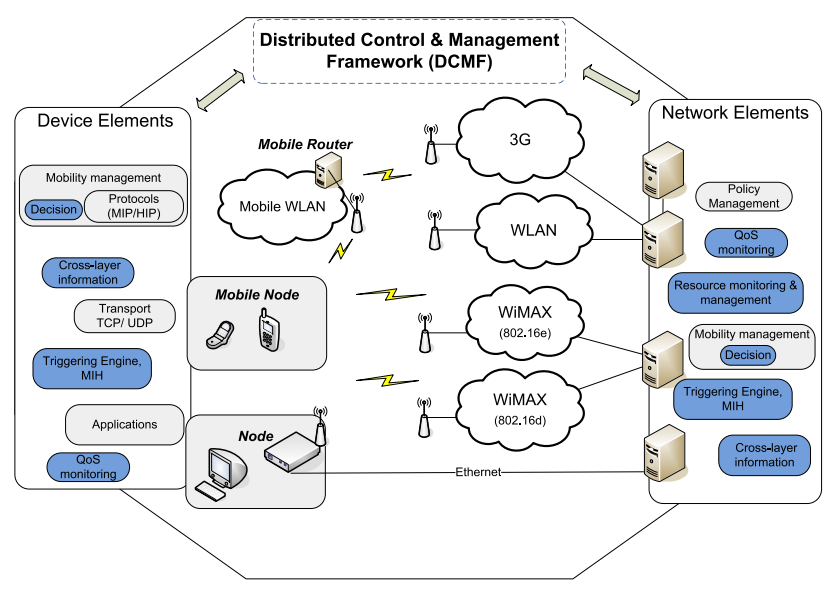

Figure 1: Illustration of the distributed control and management framework (DCMF)

The cascaded TRG functionality, that is evaluated in this paper, where TRG can act as a producer or a consumer to another TRG, is used as the starting point for building DCMF. DCMF will be built to support information change between operators, access control and different optimizations in the network architecture. And as mentioned the basis of this framework is cascaded TRG functionality that provides a unified signalling architecture between network nodes, capable of serving different entities, located either in the network or in the terminals and controlled by different players (terminals by the user, network by the service provider). The network side elements that will bene-
Table 2: Triggers provided by TRG framework

\begin{tabular}{|l|l|}
\hline Name & Description \\
\hline INTERFACE_ADD & $\begin{array}{l}\text { Network interface added to the } \\
\text { local machine }\end{array}$ \\
\hline INTERFACE_REM & $\begin{array}{l}\text { Network interface removed from } \\
\text { the local machine }\end{array}$ \\
\hline INTERFACE_CONN & $\begin{array}{l}\text { Local network interface gained IP } \\
\text { connectivity }\end{array}$ \\
\hline INTERFACE_DISC & $\begin{array}{l}\text { Local network interface lost IP } \\
\text { connectivity }\end{array}$ \\
\hline ROUTE_ADD & $\begin{array}{l}\text { Route added to the local routing } \\
\text { table }\end{array}$ \\
\hline ROUTE_DEL & $\begin{array}{l}\text { Route removed from the local } \\
\text { routing table }\end{array}$ \\
\hline WLAN_OPER_REM & $\begin{array}{l}\text { WLAN access point operational } \\
\text { status change }\end{array}$ \\
\hline WLAN_CONG_REM & $\begin{array}{l}\text { WLAN access point congestion } \\
\text { status change }\end{array}$ \\
\hline WLAN_QUAL_REM & $\begin{array}{l}\text { WLAN radio interface quality } \\
\text { status change }\end{array}$ \\
\hline
\end{tabular}

fit from such a signalling framework include, for example, policy management, network/operator aided mobility management, and resource management. On the terminal side, mechanisms such as mobility management or transport protocol optimization solutions need access to extensive amount of information related to network access characteristics and roaming.

The information that is made available through DCMF, by using the cascaded TRG functionality as a signalling between nodes, can be used for optimizing network and terminal side operations in terms of, for example, applications' Quality of Service (QoS) requirements, energy efficiency, security, and network load balancing. In order to obtain relevant information for the decision-making process, we need to rely on, for example, operator policies, QoS measurements, and various cross-layer events (e.g. those defined by IEEE 802.21). But as mentioned defining the DCMF, that will benefit of cascaded TRG functionality, is part of ongoing work.

\section{TESTBED AND EVALUATION}

We have created a testbed, which includes a 3G/UMTS cell, two WLAN access points, and one WiMAX cell (see Fig. 2). Using this testbed we show how our TRG framework can significantly improve the end-user experience. The configuration consists of three network servers, four access networks to which the mobile router (MR) is connected and a mobile client connected to the MR. For mobility the MR uses Mobile IPv4 (MIP) enhanced with the TRG framework and acts as router to the mobile network. The TRG framework provides the triggers shown in Table 2 to the MIP client running on the MR.

The decision logic added to the MIP client uses these triggers to evaluate all available networks and steer the MR to choose the best possible network. In the tests QoSMeT measurement tool [6] that is running on the streaming server and the client was used to measure the one-way QoS characteristics experienced by the video stream from the streaming server to the stream client. QoSMeT is a QoS measurement 


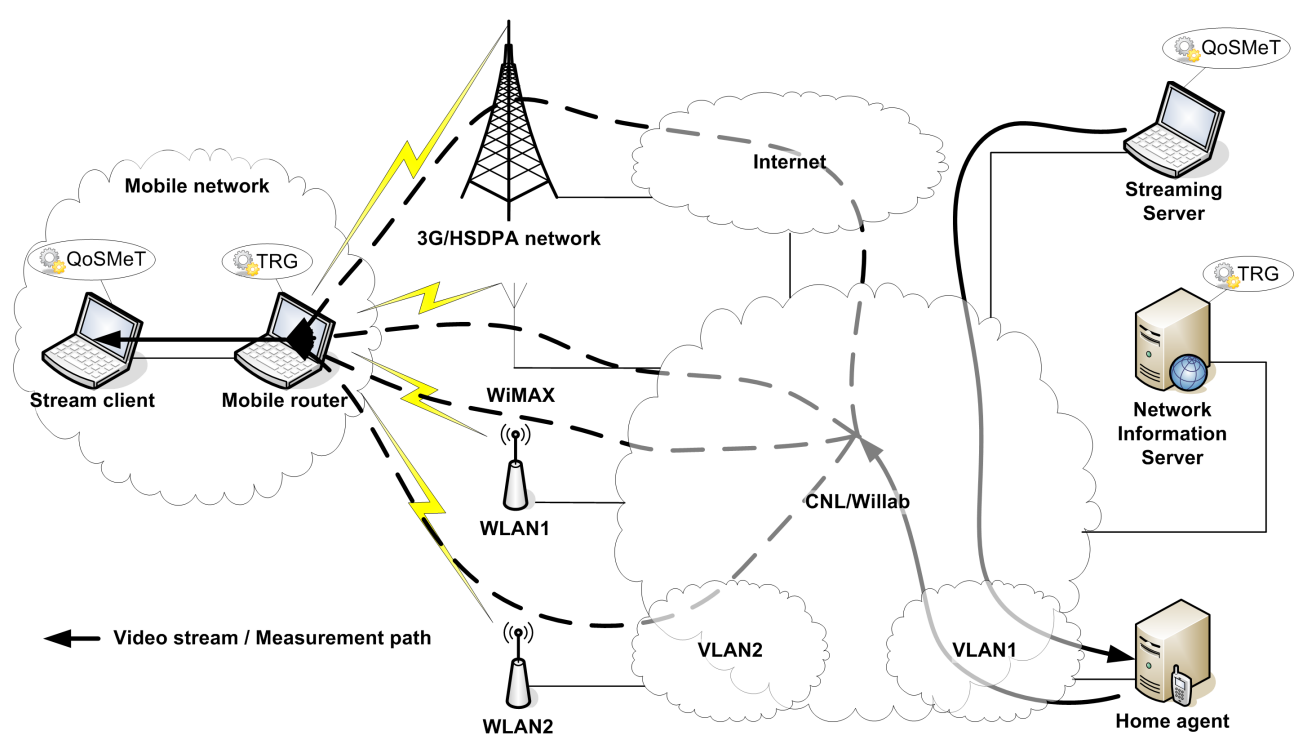

Figure 2: Movement of Mobile Node in heterogeneous environment

tool developed by VTT which uses GPS synchronisation of time in different nodes to enable measuring of one-way QoS characteristics such as packet delay or jitter of an end-toend link. The video used in the experiment was streamed as UDP packets with the size of 1358 bytes and an average bit rate of $485 \mathrm{~kb} / \mathrm{s}$.

Fig. 3 illustrates three aspects 1) the delay experienced by the video stream in our testbed with the information for triggering handovers provided by TRG, 2) the access network used for streaming the video, and 3) the vertical handovers. The MR is initially connected to the WiMAX network (A). The WiMAX network is then disconnected and the MR makes a handover to WLAN1 network (B). When WLAN1 network is congested by sending UDP traffic with a bit rate of $60 \mathrm{Mb} / \mathrm{s}$ to a another node connected to it the MR makes a handover to WLAN2 (C). Finally, WLAN2 network's transmission power is lowered gradually until the MR makes a handover to the $3 \mathrm{G}$ network (D).

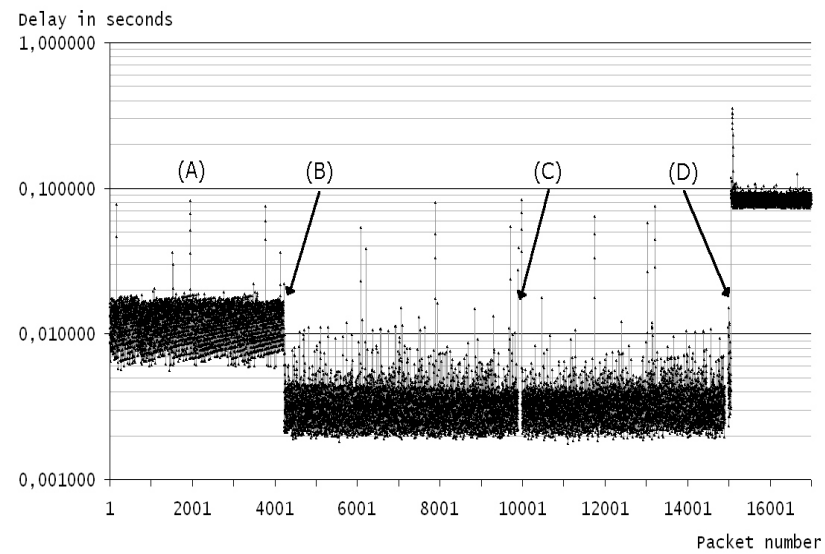

Figure 3: Handovers in test scenario
Although a network card is able to maintain connection with an access point packets can still be lost due to low signal strength. To address this the TRG framework provides triggers based on wireless network adapter signal strengths. Figure 4 illustrates a signal strength measurement by an information source in the TRG framework. The value of the signal strength has no unit as it is a value given by the network adapter driver and the thresholds must be calibrated differently for each driver. The measurement points on $\mathrm{x}-$ axis correspond roughly to seconds in time as the information source provides updates approximately once a second. When the signal strength drops below the lower threshold set in the information source it sends a trigger informing interested parties that the interface in question is considered to be down due to low signal strength. Again when the signal strength climbs above the upper threshold a trigger indicating the interface is up is sent. The interface down trigger based on the signal strength triggers the handover (D) in Figure 3.

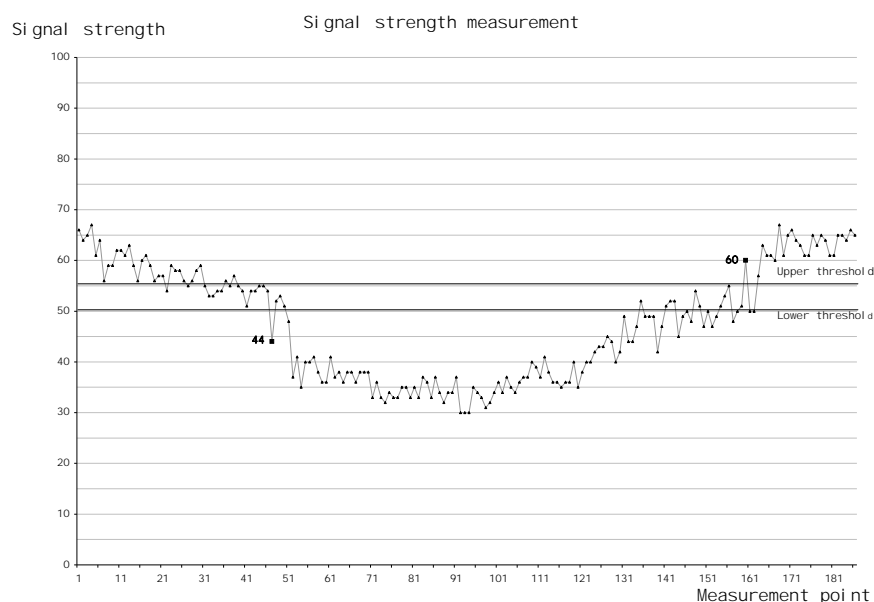

Figure 4: Signal strength measurement 


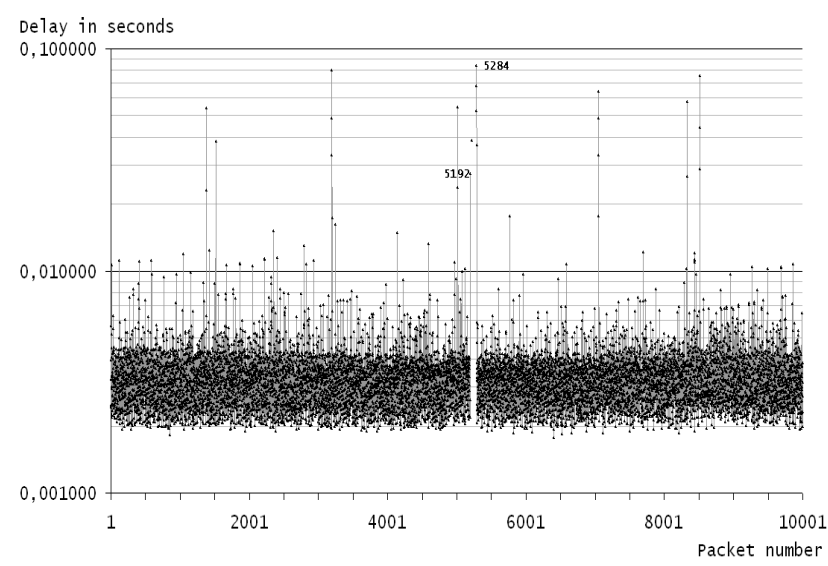

Figure 5: TRG assisted handover between two WLANs

The packet delay in the WLAN1 congestion situation ((C) in Fig.3) with the information for triggering handovers provided by TRG is graphed in Figure 5 and without the information for triggering handovers provided by TRG in Figure 6. In Figure 5 with the help of TRG the packet delay remains constant and only 90 packets are lost while the MR makes a handover to the WLAN2. Handover mechanism does not have effect to the packet loss in this case but the packets are lost because of the WLAN1 network congestion. Visually a person viewing the video experiences a small glitch in the video just before the handover is made. Figure 6 shows the loss of 6238 packets before a handover to the WLAN2 is made by the MR when there is no information provided by TRG to trigger a handover earlier. This handover is caused by the MR not being able to update its binding with its HA because of the congestion. Visually this translates to 50 seconds of the video being unintelligible during which time only occasional broken frames of the video can be displayed to a person viewing the video.

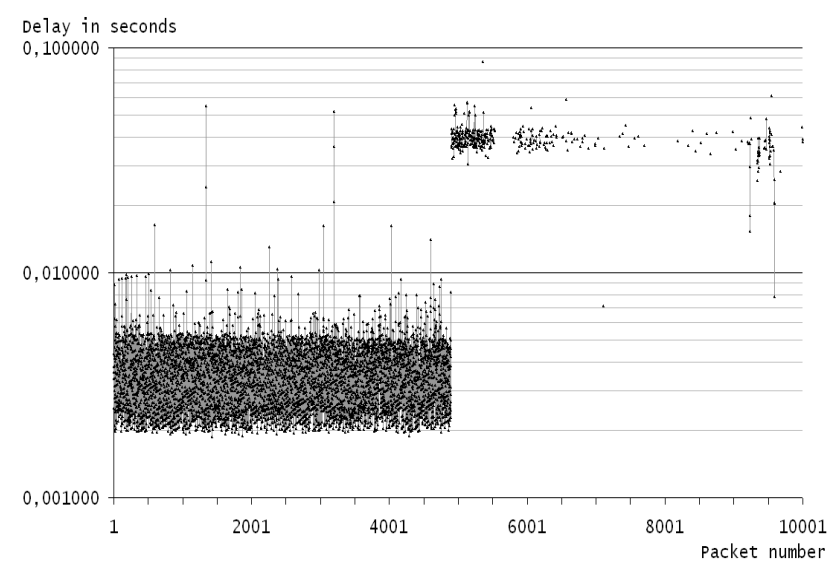

Figure 6: Handover between two WLANs without assist

The measurements without the help of the TRG frame- work were made using the default settings of the MIP client. In this case the most relevant setting being the interval at which the MR renews its binding with the HA. The default of this setting on the used MIP client is 60 seconds. This means that the MR's network connection will be down 30 seconds on average in case of a total connection loss or a severe network congestion as in the example. This problem can be dimished by lowering the binding renewal interval but this also puts unnecessary strain on the network as the communication between the MR and the HA increases. Still, with a less severe congestion it is possible for the MR to experience lowered QoS and packet losses indefinitely long periods of time as long as the MIP client is able to renew its binding with HA.

Test case evaluations, as seen from the figures, showed that there is a clear benefit when using the cascaded TRG to assist mobility management protocols. This evaluation case and the used implementations are also steps towards the media independent and seamless handovers in the mobile wireless world.

\section{RELATED WORK}

Using the event information gives benefit, for example, to proactively perform a handover in order to maintain QoS levels, keep application session continuity during handover processes. We also believe that in heterogeneous network environments several sources of events and context information should be consulted in order to achieve seamless connectivity and develop swift mobility management mechanisms like presented in [15]. Event/notification systems presented in $[16,17]$, which introduce mechanisms on how to implement such systems, along with the evaluated event generation cases are very encouraging and complementary to our effort in defining TRG together with DCMF as a specialized notification system for events which originate from the entire protocol stack. Event and notification system like TRG functionality are needed also for the future Internet solution like presented in [14]

As mentioned, the TRG functionality approach is close to the 802.21 [5] approach. The scope of the IEEE 802.21 standard is to develop a mechanism that provides link layer intelligence and other related network information to upper layers to optimize handovers between heterogeneous IEEE 802 systems and facilitates handovers between IEEE 802 and cellular systems. IEEE 802.21 helps with Handover Initiation, Network Selection and Interface Activation. The purpose is to enhance the experience of mobile device users. The standard supports handovers for both stationary and mobile users. TRG functionality is designed to provide the way to command and use information from all layers, even from the physical radio interface. Main target is to define and implement a distributed framework as discussed in section 2.2 that supports the event collection and processing, and trigger distribution possibly from hundreds of different sources. An that's also why we propose that instead of using only 802.21 services the system can consist of 802.21 services with TRG functionality as well e.g. TRG can be a distributor of MIH events for all application layer producers.

One important issue, which papers $[18,19,21]$ focus on, are ordering and time related issues considering the events. Since first of all older event should not overrun the newer or current valid event information. Desing and realization of the TRG functionality is flexible in a way that it support 
dedicated mechanisms to handle the prioritising of events or ensuring the trust between different entities, namely consumers and producers. In addition to that, TRG has a mechanism for timestamping all events as well handling the registration of producers. TRG also provides a mechanism to filter all unwanted events before disseminating them to the consumers. Current implementation provides also a way to have policies to prevent the access to the certain event information just for certain consumers.

\section{CONCLUSION}

In this paper we have concentrated to show the performance benefits gained from using a network aided information service to assist handovers in a heterogeneous networks. Triggering functional entity TRG [3], a main component of our implemented system, was used to build the distributed information service system. By using this system in a real heterogeneous environment with $3 \mathrm{G} / \mathrm{HSDPA}$, 802.11/WLAN and 802.16/WiMAX access technologies we were able to validate that our implementation is already suitable for a real network environment and test result showed that the there are real benefits gained with this system.

It needs to be noted that despite our target to provide an efficient way to handle distributed decisions and information flows within heterogeneous networks and multiaccess terminals, this system can be realized to guide the mobile terminals within a network consisting of only one access technology as well.

\section{REFERENCES}

[1] D. Johnson, C. Perkins, and J. Arkko, Mobility Support in IPv6. Request for Comments, no. 3775. IETF, June 2004.

[2] R. Moskowitz, P. Nikander, P. Jokela and T. Henderson, Host Identity protocol. Request for Comments, no. 5201. IETF, April 2008.

[3] J. Mäkelä and K. Pentikousis, Trigger Management Mechanisms. In International Symposium on Wireless Pervasive Computing, San Juan, Puerto Rico, Feb. 2007.

[4] M. Luoto and T. Sutinen, Cross-layer Enhanced Mobility Management in Heterogeneous Networks. In Proceedings of the International Conference on Communications, Beijing, China, May 2008.

[5] Media Independent Handover, IEEE Standard 802.21.

[6] J. Prokkola, M. Hanski, M. Jurvansuu, and M. Immonen, Measuring WCDMA and HSDPA Delay Characteristics with QoSMeT. InProceedings of IEEE International Conference on Communications, 2007.

[7] J. Mäkelä,Towards Seamless Mobility Support with Cross-Layer Triggering. InProceedings of Personal, Indoor and Mobile Radio Communications, 2007. PIMRC 2007.

[8] Stevens-Navarro, E.; Wong, V.W.S., Comparison between Vertical Handoff Decision Algorithms for Heterogeneous Wireless Networks. Vehicular Technology Conference, 2006. VTC 2006-Spring.

[9] Wenhui Zhang, Handover decision using fuzzy MADM in heterogeneous networks. Wireless Communications and Networking Conference,WCNC, March 2004

[10] R. Giaffreda, K. Pentikousis, E. Hepworth, R. Agero, and A. Galis, An information service infrastructure for
Ambient Networks. InProceedings of the 25th International Conference on Parallel and Distributed Computing and Networks Innsbruck, Austria, February 2007, pp. 21-27.

[11] J. Mäkelä, M.Luoto, Distributed Control and Management Framework for mobile and multi-access communication. In Proceeding of 5th International Mobile Multimedia Communications Conference, MobiMedia 2008, 7-9 July, 2008, Oulu, Finland.

[12] J. Mäkelä, K. Pentikousis, V. Kyllönen, Mobility Trigger Management: Implementation and evaluation. International Journal of Communications, Network and System Sciences, Vol.2, No.3, 2009.

[13] L. Daniel and M. Kojo, Employing Cross-layer Assisted TCP Algorithms to Improve TCP Performance with Vertical Handoffs, International Journal on Communication Networks and Distributed System, Vol. 1, No.4/5/6, 2008, pp. 433-465.

[14] C. Mingardi, G. Nunzi, D. Dudkowski, and M. Brunner, Event Handling in Clean-Slate Future Internet Management In Proceedings of the 11th IFIP/IEEE International Symposium on Integrated Network Management, IM 2009

[15] P. Vidales, J. Baliosian, J.Serrat, G. Mapp, F.Stajano, and A.Hopper, Autonomic system for mobility support in $4 \mathrm{~g}$ networks. IEEE JSAC, vol. 23, no. 12, pp. 2288-2304.

[16] R. Meier and V. Cahill, STEAM: Event-based Middleware for Wireless Ad Hoc Networks. In IEEE Int. Conf. Distributed Computing Systems (ICDCS) Workshops, 2002.

[17] H. A. Duran-Limon, G. S. Blair, A. Friday, T Sivaharan, and G. Samartzidis, A Resource and QoS Management Framework for a Real-time Event System in Mobile Ad Hoc Environments. in Int. Workshop Object-Oriented Real-Time Dependable Systems (WORDS), 2003.

[18] C. H. Lwi, H. Mohanty, and R. K. Ghosh, Causal Ordering in Event Notification Service Systems for Mobile Users. In Int. Conf. Information Technology: Coding and Computing (ITCC), 2004.

[19] E. Yoneki and J. Bacon, Determination of Time and Order for Eventbased Middleware in Mobile Peer-to-peer Environments.In IEEE Int. Conf. Pervasive Computing and Communications (PerCom) Workshops, 2005.

[20] E. Piri, T. Sutinen and J. Vehkaperä, Cross-layer Architecture for Adaptive Realtime Multimedia in Heterogeneous Network Environment. InEuropean Wireless, 2009.

[21] Dousson, C., Pentikousis K. Sutinen T., Makela, J, Chronicle Recognition for Mobility Management Triggers," Computers and Communications, InProceedings of IEEE Symposium on Computers and Communications (ISCC'07), pp.305-310, 1-4 July 2007. 